\title{
Patellar instability in Indian population: relevance of tibial tuberosity and trochlear groove distance
}

\author{
Sourabh Kulkarni, Amith P. Shetty, Karan K. Alva, Saurabh Talekar, and Vijay D. Shetty* \\ Hiranandani Orthopaedic Medical Education (HOME), Dr L. H. Hiranandani Hospital, Hillside Avenue, Hiranandani Gardens, \\ Powai, Mumbai 400076, India
}

Received 2 November 2015, Accepted 26 January 2016, Published online 25 March 2016

\begin{abstract}
Introduction: The tibial tuberosity to trochlear groove (TTTG) distance in the western population is extensively studied through various modalities such as X-rays, computerised tomography and magnetic resonance imaging. However, to our knowledge there is very little or no literature support to indicate that TTTG distance has been studied in the Indian population.

Methods: We therefore undertook a study to measure the TTTG distance in 100 MRI scans of normal Indian knees. Patients with the following co-morbidities were excluded from the study; ligamentous laxity, patellofemoral instability, mal-alignment and osteoarthritis. We measured TTTG distance on the axial MRI slices using OsiriX software. Results: The mean value for females was found to be $14.07 \mathrm{~mm}$ and that for male was found to be $13.34 \mathrm{~mm}$. Our study indicates that the TTTG distance, using MRI scans as measurement modality, in the Indian population is significantly different when compared to the published western data.

Discussion: We believe that this study can form the basis for future studies on the relationship between TTTG distance and patellar instability in Indian population.
\end{abstract}

Key words: TTTG distance, Patellar instability, Patellar dislocation.

\section{Introduction}

The tibial tuberosity to trochlear groove distance (TTTG distance) is an important parameter to determine the degree of external tibial torsion or lateralisation of the tibial tuberosity. It was initially described by Goutallier et al. in 1978 on axial radiographs taken with the knee in 30 degrees of flexion and neutral rotation [1]. It is known that excessive tibial torsion can result in patellar maltracking and that a TTTG distance greater than $20 \mathrm{~mm}$ can lead to patellar instability [2]. TTTG distance in the western population is extensively studied through various modalities such as X-rays, computerised tomography and magnetic resonance imaging (MRI). However to our knowledge, there is very little or no literature support to indicate that TTTG distance has been studied in the Indian population. We therefore undertook a study to measure the TTTG distance in 100 MRI scans of normal skeletally matured Indian knees.

\section{Material and methods}

We reviewed MRI scans of 100 skeletally matured knees in the Indian population. We excluded MRI scans showing any

\footnotetext{
*Corresponding author: vijaydshetty@gmail.com
}

ligamentous injury or any internal derangement of the knee, other than meniscal injury. We also excluded MRI scans of those individuals who, on clinical examination, showed signs and symptoms of patellofemoral instability, ligamentous laxity, malalignment or osteoarthritis as this may give abnormal measurement of the TTTG distance. The MRI scans were done with the individual in the supine position and the knee in full extension. Each measurement of TTTG distance was done by one consultant radiologist and one orthopaedic surgeon.

\section{Measurement of TTTG distance}

All measurements were done by using 1.5 tesla MRI scan. Only axial views were used to measure the TTTG distance in all individuals. We measured the TTTG distance by calculating the horizontal distance between the vertical line passing through the apex of the tibial tuberosity and the vertical line passing through the apex of the trochlear groove as described by Wittstein et al. [3] and Pandit et al. [4]. To avoid interobserver bias, four readings were taken on each MRI study by two observers. All readings were done using OsiriX software, which runs on Macintosh system (Apple Inc. Cupertino, USA).

To start with the measurement, we first selected an axial image with the deepest trochlear groove (Figure 1a). On this 
(a)

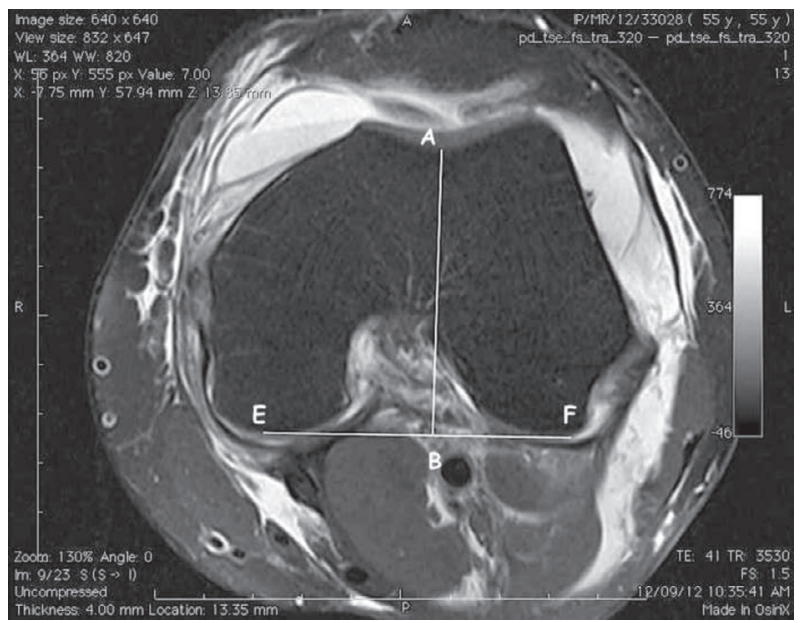

(b)

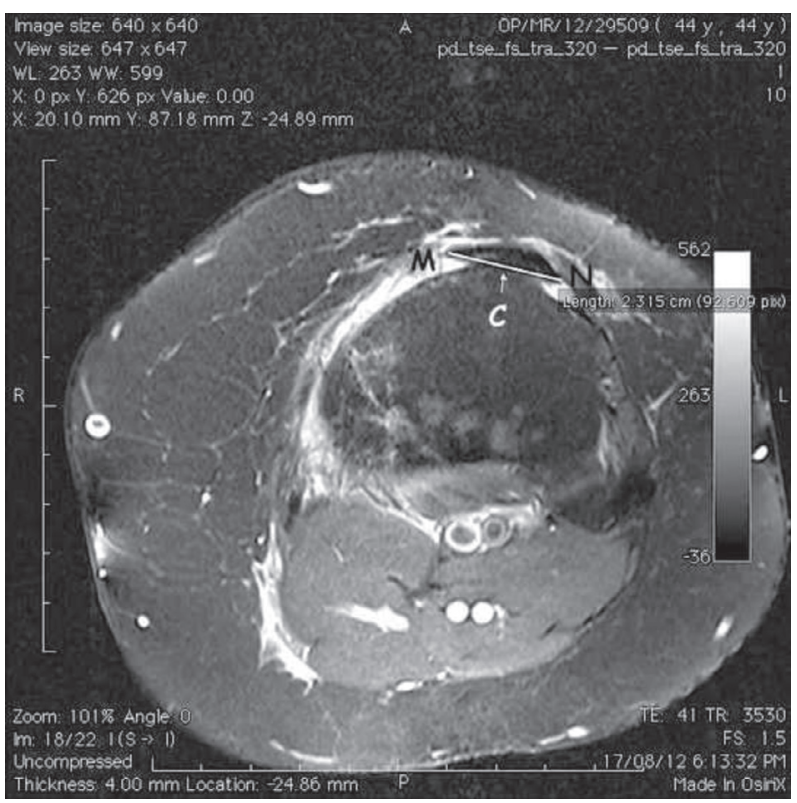

(c)

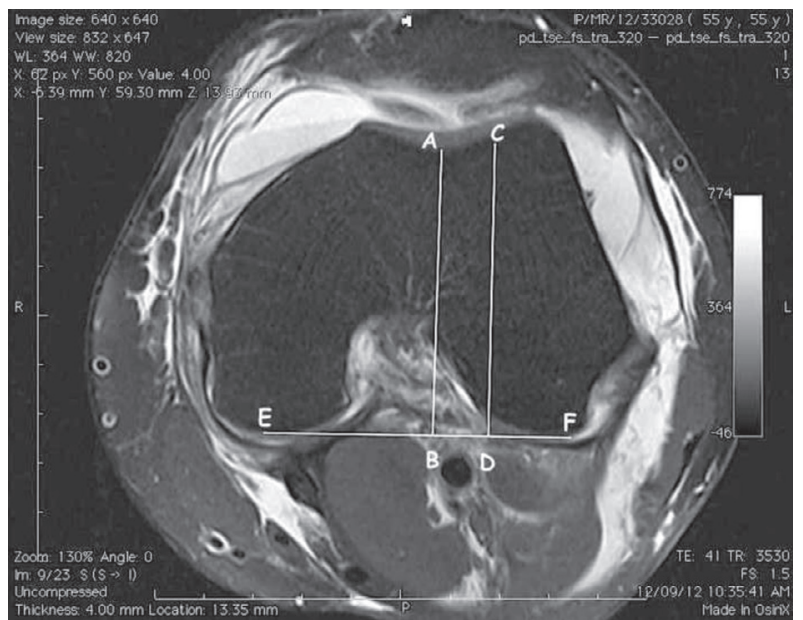

Figure 1. Measurement of TTTG distance using OsiriX software.

image a posterior condylar line (Line EF) is drawn. A perpendicular line is drawn from the deepest point of the trochlear
Table 1. Age and TTTG distance characteristics.

\begin{tabular}{llcc}
\hline Sex & & Age in years & TTTG distance on MAC \\
\hline $\mathrm{F}$ & $N$ & 40 & 40 \\
& Minimum & 22 & 6.25 \\
& Maximum & 61 & 19.84 \\
& Range & 39 & 13.59 \\
& Mean & 39.20 & 14.07 \\
& Median & 37.00 & 14.01 \\
& Std. deviation & 9.45 & 3.03 \\
& Std. error of mean & 1.49 & 0.48 \\
M & 60 & 60 \\
& Minimum & 20 & 6.15 \\
& Maximum & 60 & 21.78 \\
& Range & 40 & 15.63 \\
& Mean & 34.77 & 13.19 \\
& Median & 32.00 & 13.34 \\
Std. deviation & 11.27 & 3.14 \\
Std. error of mean & 1.46 & 0.41 \\
$N$ & 100 & 100 \\
Total & 20 & 6.15 \\
& Minimum & 61.78 \\
Maximum & 41 & 15.63 \\
Range & 36.54 & 13.54 \\
Mean & 34.50 & 13.54 \\
Median & 10.75 & 3.11 \\
Std. deviation & 1.08 & 0.31 \\
Std. error of mean & &
\end{tabular}

For TTTG: $-t$ test $=-1.394, p=0.166$, not significant.

groove (point A) to line EF in Figure 1a. The images were then scrolled down to the tibial tuberosity until reaching a slice showing the tibial tuberosity with the distal most part of the patellar tendon attached to it (Figure 1b). On this slice, the boundaries of the patellar tendon (Line MN, Figure 1b) were marked and the midpoint of this line (point $\mathrm{C}$ ) represented the tibial tuberosity.

The cursor was kept steady on point $\mathrm{C}$ and then images were scrolled up to the previous slice on which markings were done. From the point on which cursor was placed a second perpendicular was drawn on the posterior condylar line (Line CD) as shown in Figure 1c. The distance between these two perpendiculars (distance BD) is calculated as the TTTG distance.

\section{Statistical analysis}

The statistical analysis was carried out using SPSS 16.0 and MS Excel 2007. Statistical analyses tested the null hypotheses of no differences in patients with two groups of different sexes at $95 \%$ significance level. A $p$ value of less than 0.05 was considered significant $(p<0.05)$. Chi square test and $t$ test were also used in the statistical analysis. We applied t test to compare the results of our study with other two studies.

\section{Results}

Table 1 shows demographic data. The coefficient of variation was $<10 \%$ for both intra and inter-observer analysis. Of the 100 MRI scans, 60 knees were of males and 40 were of females. The mean age of the study group was 37 years (range 
Table 2. Comparison of all MRI studies.

\begin{tabular}{llll}
\hline & \multicolumn{1}{c}{ Study 1 } & \multicolumn{1}{c}{ Study 2 } & \multicolumn{1}{c}{ Study 3 } \\
\hline Author & Pandit et al. & Wittstein et al. & Our study \\
Year & 2011 & 2006 & 2012 \\
Sample size & 100 & 20 & 100 \\
$\begin{array}{l}\text { Mean TTTG } \\
\quad \text { distance }\end{array}$ & 10 & 9.4 & 13.5 \\
$S D$ & 0.5 & 0.3 & \\
$t$ value* & 11.15 & 12.93 & - \\
$p$ value* & $<0.001$ & $<0.001$ & - \\
Significance* & $\begin{array}{c}\text { Highly } \\
\text { significant }\end{array}$ & $\begin{array}{c}\text { Highly } \\
\text { significant }\end{array}$ \\
\hline
\end{tabular}

${ }^{*}$ In comparison with our study.

20-61 years). The mean TTTG distance was found to be $13.54 \pm 6.22 \mathrm{~mm}$ (range 7.32-19.76 mm). The mean value for females was found to be $14.07 \pm 6.06 \mathrm{~mm}$ (range $8.01-20.13 \mathrm{~mm}$ ) and that for males was found to be $13.34 \pm 6.28 \mathrm{~mm}$ (range 7.06-19.62 mm; $p=0.166$, not significant). Our mean values, when compared to published data $[3,4]$, were found to be significantly different statistically $(p=0.001)$ (Table 2).

\section{Discussion}

The position of the tibial tubercle is crucial for normal functioning of the quadriceps mechanism because it decides the direction of the inferolateral force vector on the patella and pull of the quadriceps mechanism. Normally, the tibial tuberosity is placed more in line under the femoral sulcus, hence the inferior force vector is much more in magnitude than the lateral force vector. This prevents the lateral subluxation of the patella. But as the tibial tuberosity is placed more laterally, the lateral force vector increases in magnitude, which tries to subluxate or dislocate the patella laterally.

The TTTG distance is one of the major parameters to determine the lateralisation of tibial tuberosity and degree of external tibial torsion. TTTG distance plays a major role in the assessment of patellar instability [5]. It is also used as an indication for a distal realignment procedure for patellar instability [6]. Another factor affecting patellar instability is patella alta. The patellar height can be measured by various methods, the most common being Caton-Deschamps index [7]. Here the distance between the lower border of the articular surface of the patella and the antero-superior border of the tibia is compared with the length of the patellar articular cartilage.
The TTTG distance can be measured accurately with both computerised tomography as well as MRI; however, an MRI scan study has several advantages over CT scan study as it is free of radiation hazards and the MRI scan can evaluate the cartilage damage as a result of recurrent patellar dislocations [8]. In patients with severe trochlear dysplasia requiring trochleoplasty, assessment of the cartilage is pivotal as pre-operative cartilaginous degeneration has been associated with inferior results $[5,9,10]$. MRI has an added advantage of helping in determining the exact centre of patellar tendon attached to tibial tuberosity $[11,12]$.

There is a wide range of normal TTTG values given in different studies. Using the MRI scan as an imaging modality Wittstein et al. [3] found it as $9.4 \pm 0.6 \mathrm{~mm}$ and Pandit et al. [4] found it as $9.91 \mathrm{~mm}$ in males and $10.04 \mathrm{~mm}$ in females. Table 3 shows these studies with their results.

In our study, the mean normal value of the TTTG distance in an adult Indian population was found to be $13.54 \pm 6.22 \mathrm{~mm}$ (males 13.19 $\pm 6.28 \mathrm{~mm}$ and females $14.07 \pm 6.06$ ). The difference between the values for males and females was not found to be statistically significant $(p=0.166)$.

As stated in Table 3, we found two studies that used MRI scan as an imaging modality. After comparing our study results with their results we found that the difference in the TTTG distances is statistically significant in our study. Our values indicate that the TTTG distance is far above the values published in the literature. However, it is not clear whether the incidence of patellar dislocation, in the Indian population, is different from that of western population. In our study we used double blindedness of the measurements between two investigators. We also had a uniform method of measurement.

\section{Conclusion}

Our study indicates that TTTG distance, using MRI scans as a measurement modality, in the Indian population is significantly different when compared to the published western data. We do not yet know whether this has an impact on the incidence of patellar instability in the Indian population. We believe that this study forms the basis for further research to study the association between TTTG distance and patellar instability in Indian knees.

\section{Conflict of interest}

The authors declare no conflict of interest in relation with this paper.

Table 3. Studies showing TTTG distance measurement.

\begin{tabular}{lcccccc}
\hline Author & Year & Modality & Sample size & Mean TTTG distance & $\begin{array}{c}\text { TTTG distance } \\
\text { for males }\end{array}$ & $\begin{array}{c}\text { TTTG distance } \\
\text { for females }\end{array}$ \\
\hline $\begin{array}{c}\text { Pandit, Frampton, } \\
\quad \text { Stoddart and Lynskey }\end{array}$ & 2011 & MRI & 100 & $10 \pm 1 \mathrm{~mm}$ & $9.91 \mathrm{~mm}$ & $10.04 \mathrm{~mm}$ \\
$\begin{array}{c}\text { Wittstein, Bartlett, } \\
\text { Easterbrook and Byrd }\end{array}$ & 2006 & MRI & 20 & $9.4 \pm 0.6 \mathrm{~mm}$ & $(95 \%$ CI 8.9-10.8 mm) & $(95 \% \mathrm{CI} 8.9-11.1)$ \\
\hline
\end{tabular}




\section{References}

1. Goutallier D, Bernageau J, Lecudonnec B (1978) The measurement of the tibial tuberosity. Patella groove distanced technique and results. Rev Chir Orthop Reparatrice Appar Mot 64(5), 423-428.

2. Dejour H, Walch G, Nove-Josserand L, Guier C (1994) Factors of patellar instability: an anatomic radiographic study. Knee Surg Sports Traumatol Arthrose 2(1), 19-26.

3. Wittstein J, Bartlett E, Easterbrook J, Byrd J (2006) Magnetic resonance imaging evaluation of patellofemoral malalignment. Arthroscopy 22(6), 643-649.

4. Pandit S, Frampton C, Stoddart J, Lynskey T (2011) Magnetic resonance imaging assessment of tibial tuberosity-trochlear groove distance: normal values for males and females. Int Orthop 35(12), 1799-1803.

5. Schöttle P, Fucentese S, Pfirrmann C, Bereiter H, Romero J (2005) Trochleaplasty for patellar instability due to trochlear dysplasia. Acta Orthopaedica 76(5), 693-698.

6. Schöttle P, Fucentese S, Romero J (2005) Clinical and radiological outcome of medial patellofemoral ligament recon- struction with a semitendinosus autograft for patella instability. Knee Surg Sports Traumatol Arthrosc 13(7), 516-521.

7. Caton J (1989) Method of measuring the height of the patella. Acta Orthop Belg 55, 385-386.

8. Schoettle P, Zanetti M, Seifert B, Pfirrmann C, Fucentese S, Romero J (2006) The tibial tuberosity-trochlear groove distance; a comparative study between CT and MRI scanning. Knee 13(1), 26-31.

9. DeJour D, Saggin P (2010) The sulcus deepening trochleoplasty the Lyon's procedure. Int Orthop 34(2), 311-316.

10. Utting M, Mulford J, Eldridge J (2008) A prospective evaluation of trochleoplasty for the treatment of patellofemoral dislocation and instability. J Bone Jt Surg $\mathrm{Br}$ vol 90-B(2), 180-185.

11. Maquet P (1976) Advancement of the tibial tuberosity. Clin Orthop Relat Res 115, 225-230.

12. Feller JA, Amis AA, Andrish JT, Arendt EA, Erasmus PJ, Powers CM (2007) Surgical biomechanics of the patellofemoral joint. Arthroscopy 23(5), 542-553.

Cite this article as: Kulkarni S, Shetty AP, Alva KK, Talekar S \& Shetty VD (2016) Patellar instability in Indian population: relevance of tibial tuberosity and trochlear groove distance. SICOT J, 2, 14 